\title{
Robótica na Educação: Uma Revisão Sistemática dos Últimos 10 Anos
}

\section{Ranulfo Plutarco Bezerra Neto ${ }^{1}$, Diego Porto Rocha ${ }^{1}$, André Macêdo Santana ${ }^{1}$, Anderson Abner de Santana Souza ${ }^{2}$}

\author{
${ }^{1}$ Departamento de Computação - Universidade Federal do Piauí (UFPI) \\ Caixa Postal 64049-550 - Teresina - PI - Brasil \\ ${ }^{2}$ Departamento de Informática - Universidade do Estado do Rio Grande do Norte (UERN) \\ Caixa Postal 59080-100 - Capim Macio - RN - Brasil \\ \{ranulfo0s, diego.specter, and.abner\}@gmail.com, andremacedo@ufpi.edu.br
}

\begin{abstract}
This paper goal is to present a systematic revision of the works related to robotic on education, which were published on three national events of this area: Informatics on Education Brazilian Symposium (SBIE), Informatics on School Workshop (WIE), Robotics on Education Workshop (WRE), between 2004 to 2014. The results shown an increasing interest of brazilian community for the area, highlighting the northeast and southeast regions of Brazil responsible for most of the works. It was also found that $63 \%$ of this works represents proposals of the use of robotics on elementary school with new methodologies and robots to assist pedagogy in the use of robotics.
\end{abstract}

Resumo. Este artigo tem como objetivo apresentar uma revisão sistemática de literatura dos trabalhos relacionados à robôtica na educação, publicados em três importantes eventos nacionais na área, Simpósio Brasileiro de Informática na Educação (SBIE), Workshop de Informática na Escola (WIE) e Workshop de Robôtica na Educação (WRE), entre os anos de 2004 e 2014. Os resultados obtidos mostram um aumento crescente no interesse da comunidade brsileira na área, destacando-se as regiões Nordeste e Sudeste do Brasil responsáveis por grande parte das pesquisas. Foi encontrado na 63\% dos artigos, propostas do uso da robótica no ensino fundamental, com novas metodológias ou tipos de robôs para auxiliar a pedagogia no uso da robótica.

\section{Introdução}

De acordo com [Monarcha 1999], a pedagogia é uma ciência autônoma e, por meio dela, gera um corpo de conhecimentos sem o qual lhe seria impossível qualquer construção de modelos educativos. Robótica pedagógica ou robótica educacional é um exemplo de construção de modelo educativo inovador e eficaz em sua proposta.

O modelo em questão consiste na criação de ambientes de aprendizagem que reúnem materiais de montagem compostos por peças diversas, interativas e controláveis por computador através de softwares que permitam programar o funcionamento dos protótipos montados. Essa interação cria uma atmosfera de interesse e envolvimento por parte dos alunos, podendo integrar-se com outras áreas do conhecimento, desenvolvendo atividades no âmbito interdisciplinar, no qual os estudantes vivenciam de forma prática conceitos anteriormente vistos em sala de aula. 
A robótica educacional (RE) é um paradigma recente. Os primeiros conceitos desse padrão surgiram com [Papert 1986] em meados do ano de 1986 nos Estados Unidos. No cenário brasileiro, a RE começou a ser desenvolvida na segunda metade dos anos de 1990, pelas instituições de ensino UFRJ, UNICAMP e UFRGS, em conformidade com [d'Abreu 2014]. Por se tratar de um conceito singular, recente e não-recorrente, as suas aparições na literatura ainda são muito escassas, dificultando o aprofundamento e a popularização desses sistema tecnológico.

Dessa forma, foi usado como motivação a deficiência de literatura especifica na área para desenvolvermos uma revisão sistemática sobre a utilização da robótica no âmbito educacional brasileiro. A pesquisa foi realizada com base em artigos do SBIE e WIE, datados entre 2004 e 2014 e artigos do WRE datados entre 2012 e 2014. Nessa revisão sistemática respondemos questões importantes para a elucidação do quadro contemporâneo da RE no Brasil.

\section{Método}

Segundo [Kitchenham 2007], uma Revisão Sistemática de Literatura (RSL) é efetuada para buscar e avaliar quantitativa e qualitativamente os estudos já efetuados na área que tenham sido publicados ou disponibilizados para a comunidade científica e que tenham determinada importância acadêmica como base de pesquisas. Para isso, é necessário determinar questões de pesquisa, e, a partir destas questões, realizar o levantamento dos dados essencias para respondê-las, orientando assim a leitura e seleção dos artigos de modo a selecionar os que farão parte da RSL. É indispensável, portanto, estabelecer critérios de inclusão e exclusão antes de realizar a pesquisa. A partir das questões formuladas e destes critérios, foi desenvolvido o protocolo utilizado como guia durante todo o RSL para a coleta de evidências.

\subsection{Questões de Pesquisa}

Uma das etapas essenciais desta RSL foi a definição das questões de pesquisa que conduziram a busca dos documentos relevantes com o objetivo de responder a questão central do estudo: "Como se caracteriza o uso da robótica no ensino e como ela é distribuída no Brasil?". Através do suporte de quatro especialistas da área a questão principal foi traduzida nas seguintes questões de pesquisa:

- Q1 - Quais são as instituições de pesquisa envolvidas nesta área e como elas estão distribuídas pelo Brasil?

- Q2 - Quais as áreas do conhecimento que estão sendo exploradas por esta temática e para que nível de escolaridade estas pesquisas estão sendo destinadas?

- Q3 - Quais as características dos robôs utilizados?

- Q4 - Quantos trabalhos são reflexões, quantos são propostas e quantos apresentam experiências?

\subsection{Estratégia e Processo de Busca}

A procura de artigos científicos no Brasil é dificultada pela ausência de uma plataforma de busca, por conseguinte, parte das práticas abordadas por [Kitchenham 2007] não foram possíveis de serem aplicadas. Além disso, deparamo-nos com impossibilidade de encontrar anais do WRE entre os anos 2010 e 2011. 
A seleção inicial foi realizada baseando-se em todos os artigos publicados de SBIE, WRE e WIE dos anos de 2004 a 2014, cujos títulos e resumos foram encontradas as palavras robô e robótica. A partir desta seleção todos os artigos foram lidos e aplicados os critérios de exclusão e inclusão.

\subsection{Critérios de Inclusão/Exclusão}

A inclusão dos trabalhos selecionados foi definida como a capacidade do artigo de responder, pelo menos, uma das questões de pesquisa. Após o processo de inclusão o processo de exclusão foi feito com as seguintes diretrizes:

- Artigos que abordam robótica, porém não há aplicação na educação.

- Artigos que não foram escritos no Brasil

- Artigos que abordam agentes como forma de robótica

\section{Resultados}

Esta seção apresenta os resultados encontrados na RSL obtido a partir dos estudos primários selecionados e uma breve análise destes artigos.

\subsection{Informações Gerais}

Ao término da seleção e análise dos artigos foram encontrados 1537 trabalhos nos congressos: WIE, SBIE e WRE. Deste universo, apenas 65 artigos se enquadraram nas políticas de inclusão e exclusão descritas anteriormente, dentre os anos de 2004 e 2014, como pode ser visto na Tabela 1:

Tabela 1. Resultado geral das buscas para SBIE, WIE e WRE.

\begin{tabular}{|c|c|c|c|}
\hline Evento & Artigos Incluídos & Total de Artigos & $\begin{array}{c}\text { Artigos Incluidos / } \\
\text { Total de Artigos (\%) }\end{array}$ \\
\hline SBIE & 18 & 999 & 1.80 \\
\hline WIE & 14 & 478 & 2.92 \\
\hline WRE & 33 & 60 & 55 \\
\hline Total & $\mathbf{6 5}$ & $\mathbf{1 5 3 7}$ & $\mathbf{4 . 2 2}$ \\
\hline
\end{tabular}

Como pode ser visto na Tabela 1, o Workshop de Robótica na Educação, com apenas 3 anais encontrados na literatura, se destaca pelo maior número de artigos incluídos na pesquisa. Dado que os artigos aceitos pelo WRE tem o mesmo foco do nosso tema, é compreendido o grande volume de trabalhos classificados na RSL. A Figura 1(a) ilustra a distribuição de trabalhos encontrados entre os três congressos.

A Figura 1(b) ilustra a evolução da abordagem da RE, identificada pelo processo de seleção, ao longo dos anos. A influencia do WRE na evolução da RE é marcante na Figura 1(b), visto que seus artigos encontrados estão entre os anos de 2012 e 2014.

\subsection{Respostas às Questões de Pesquisa}

Nesta seção serão respondidas as questões de pesquisa, com base nos estudos feitos. 


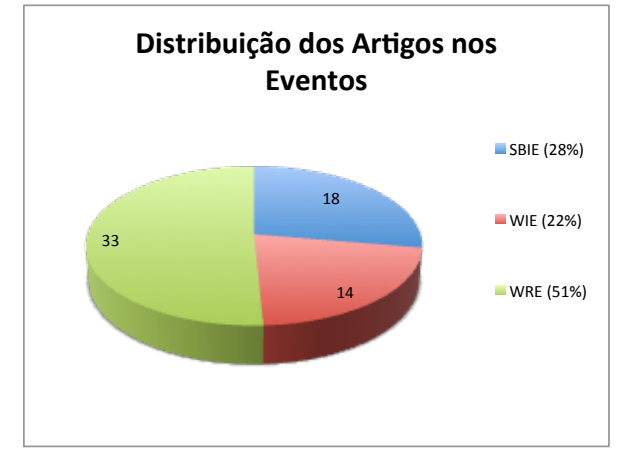

(a)

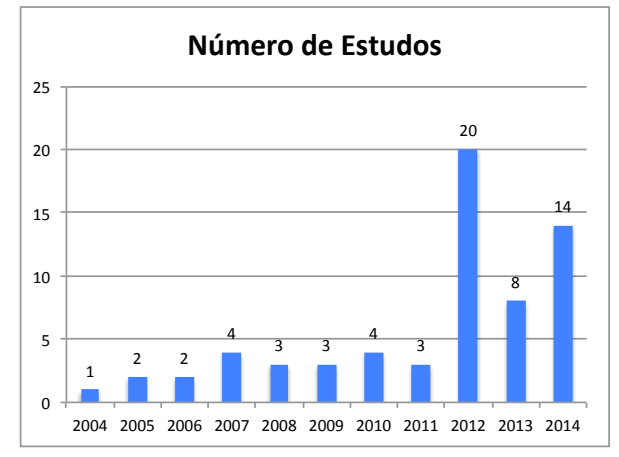

(b)

Figura 1. Distribuição dos Artigos. (a): Por Evento. (b): Por Ano (2004 a 2014)

\subsubsection{Q1: Quais são as instituições de pesquisa envolvidas nesta área e como elas estão distribuídas pelo Brasil?}

Após a análise da quantidade de artigos por instituição, foi contabilizado 41 instituições que publicaram trabalhos referentes a RE no Brasil. A Tabela 2 consta todas as instituições por região do Brasil com a respectiva quantidade de artigos publicados. É importante ressaltar que há 7 artigos em parceria com mais de uma instituição, portanto o somatório da quantidade de artigos da Tabela 2 excede o total de artigos selecionados por esta RSL. A Tabela 2 contém mais detalhes.

Ao realizar uma análise geográfica dos artigos, foi constatado que os estudos da RE são concentrados nas regiões nordeste, sudeste e sul, com a incidência de apenas três trabalhos nas regiões centro-oeste e norte, também foi observado um grande número de instituições com no máximo três publicações nesta área, com exceção da Universidade Federal do Rio Grande no Norte (UFRN) que lidera com onze artigos a quantidade de publicações por instituição.

Como pode ser visto na Figura 2 foram encontrados 24 artigos da região nordeste sendo 5 (SBIE), 5 (WIE) e 14 (WRE). Na região sudeste foram encontrados 26, sendo 5 (SBIE), 8 (WIE) e 13 (WRE). Na região sul foram encontrados 12 artigos, sendo 6 (SBIE), 1 (WIE) e 5 (WRE). Na região centro-oeste foi encontrado 1 artigo no evento WRE. E por fim na região norte foram encontrados 2 artigos do evento SBIE.

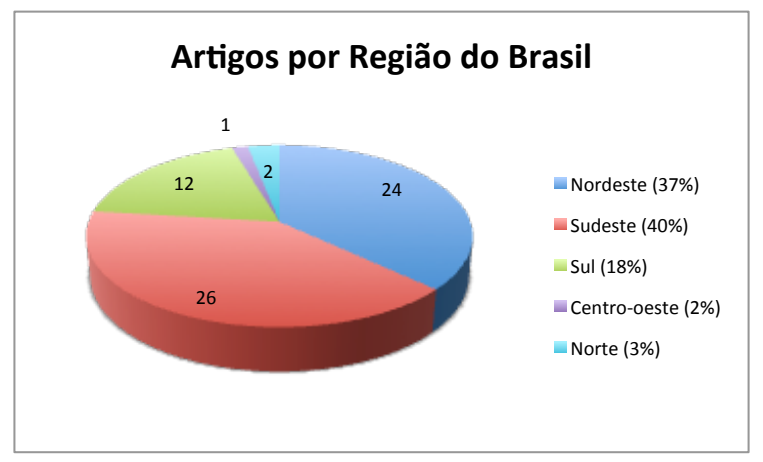

Figura 2. Distribuição dos artigos pelas regiões do Brasil 
Tabela 2. Resultado geral das buscas para SBIE, WIE e WRE.

\begin{tabular}{|l|l|l|}
\hline \multicolumn{1}{|c|}{ Região } & $\begin{array}{c}\text { Instituição } \\
\text { dos } \\
\text { Artigos }\end{array}$ & $\begin{array}{c}\text { Qtde } \\
\text { Artigos }\end{array}$ \\
\hline Nordeste & (IFBA) & 1 \\
\hline \multirow{5}{*}{} & (IFPB) & 1 \\
\cline { 2 - 3 } & (UEM) & 2 \\
\cline { 2 - 3 } (UEPB) & 1 \\
\cline { 2 - 3 }
\end{tabular}

\begin{tabular}{|l|l|}
\hline $\begin{array}{c}\text { Instituição } \\
\text { dos } \\
\text { Artigos }\end{array}$ & $\begin{array}{c}\text { Qtde } \\
\text { Artigos }\end{array}$ \\
\hline (UFAL) & 1 \\
\hline (UFBA) & 1 \\
\hline (UFMA) & 1 \\
\hline (UFPB) & 2 \\
\hline
\end{tabular}

\begin{tabular}{|l|l|l|}
\hline Sudeste & (CPTI) & 1 \\
\hline \multirow{5}{*}{} & (FAETERJ) & 1 \\
\cline { 2 - 3 } & (IFES) & 2 \\
\cline { 2 - 3 } & (IFMG) & 2 \\
\cline { 2 - 3 } & (IME) & 2 \\
\cline { 2 - 3 }
\end{tabular}

\begin{tabular}{|l|l|l|}
\hline Sul & $($ UTFPR) & 1 \\
\hline \multirow{4}{*}{} & (UFRG) & 1 \\
\cline { 2 - 3 } & (IERGS) & 1 \\
\cline { 2 - 3 } & (IFSUL) & 1 \\
\hline
\end{tabular}

\begin{tabular}{|l|l|l|}
\hline Norte & (UFAM) & 1 \\
\hline
\end{tabular}

\begin{tabular}{|l|l|}
\hline (ITA) & 1 \\
\hline (UFABC) & 1 \\
\hline (UFES) & 2 \\
\hline (UFF) & 2 \\
\hline (UFRJ) & 3 \\
\hline
\end{tabular}

\begin{tabular}{|l|l|}
\hline (RME) & 1 \\
\hline (UFFS) & 2 \\
\hline (UFRGS) & 3 \\
\hline (ULBRA) & 2 \\
\hline
\end{tabular}

\begin{tabular}{l|l|}
\hline (UFRR) & 1 \\
\hline
\end{tabular}

\begin{tabular}{|l|l|}
\hline $\begin{array}{c}\text { Instituição } \\
\text { dos } \\
\text { Artigos }\end{array}$ & $\begin{array}{c}\text { Qtde } \\
\text { Artigos }\end{array}$ \\
\hline (UFRN) & 10 \\
\hline (UFS) & 2 \\
\hline (FSS) & 1 \\
\hline UFRB & 1 \\
\hline
\end{tabular}

\begin{tabular}{|l|l|}
\hline (UFSJ) & 2 \\
\hline (UFU) & 1 \\
\hline (UNESP) & 3 \\
\hline (UNICAMP) & 3 \\
\hline (USP) & 3 \\
\hline
\end{tabular}

\begin{tabular}{|l|l|}
\hline (UNISC) & 2 \\
\hline (UNOESC) & 1 \\
\hline (UPF) & 1 \\
\hline
\end{tabular}

\begin{tabular}{|l|l|l|}
\hline Centro-oeste & (UEG) & 1 \\
\hline
\end{tabular}

\subsubsection{Q2 - Quais as áreas do conhecimento que estão sendo exploradas por esta temática e para que nível de escolaridade estas pesquisas estão sendo destina- das?}

A robótica vem com uma base sólida que é também utilizada para o desenvolvimento da interdisciplinaridade (dentro da robótica) servindo como ferramenta eficiente neste conceito de estudo. Como pode ser visto na Figura 3(a) é mostrado que a robótica ou o ensino de robótica contribui para o amadurecimento dos conhecimentos de diversas matérias não só como matemática, geometria, física, mas também auxiliando no ensino de ciências, história e geografia como pode ser visto em [de Castro Pinto et al. 2012] e [Benitti et al. 2009].

Dentre os artigos pesquisados a maior porcentagem quantitativa do ensino de robótica se situa no nível de escolaridade do ensino fundamental, abrangendo um total de 63\% dos artigos que trouxeram o nível de escolaridade especificados dentro de sua pesquisa. Seguido do fundamental os níveis com maior relevância são: Ensino Médio(29\%) e Ensino Superior(25\%), que juntos ultrapassam metade das pesquisas realizadas. É importante salientar que em certos artigos mais de um nível de escolaridade é abordado. Mais detalhes podem ser observados na Figura 3(b) 
CBIE-LACLO 2015

Anais do XXVI Simpósio Brasileiro de Informática na Educação (SBIE 2015)

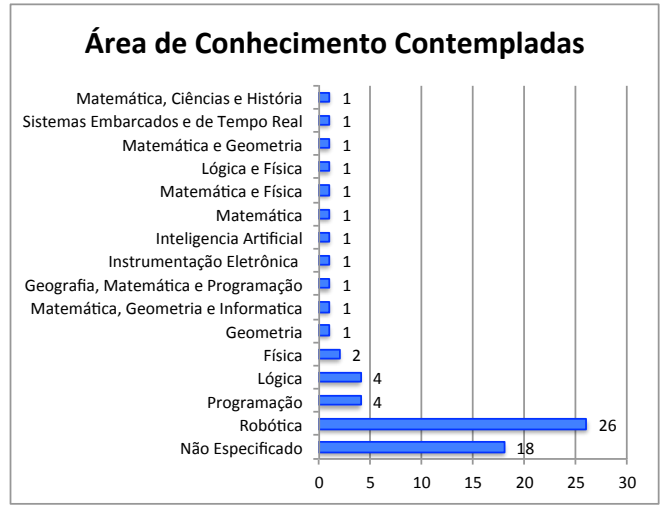

(a)

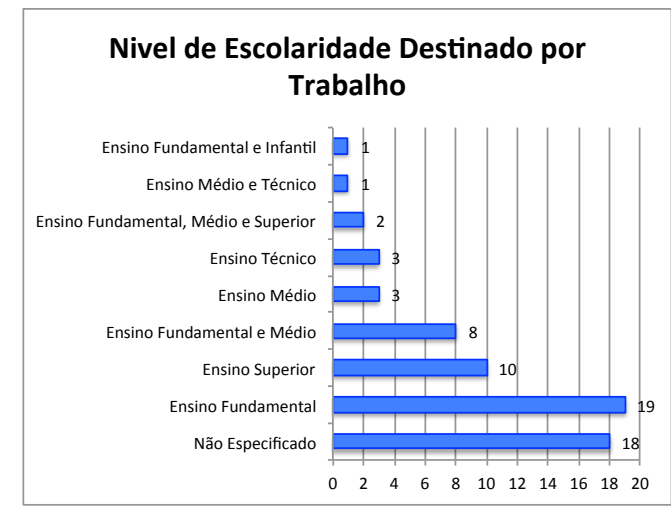

(b)

Figura 3. Distribuição dos Trabalhos. (a): Por área de Conhecimento Contempladas. (b): Por Nível de Escolaridade Destinado.

\subsubsection{Q3 - Quais as características dos robôs utilizados?}

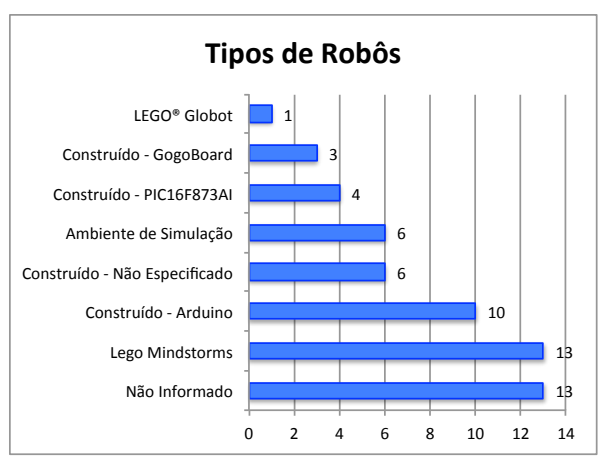

(a)

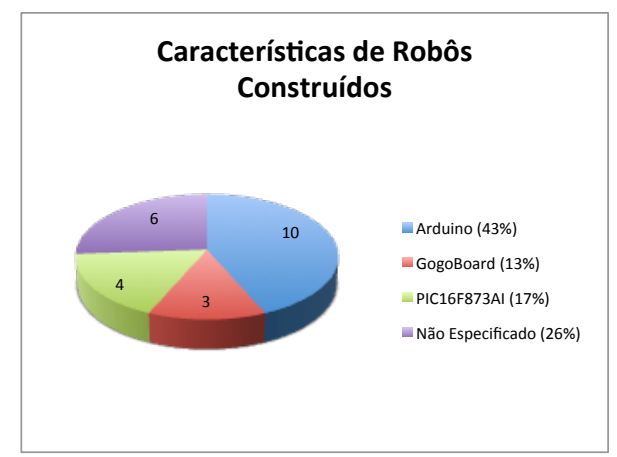

(b)

Figura 4. Distribuição dos Robôs. (a): Em Geral. (b): Apenas Construídos

Tratando-se dos tipos de robôs utilizados nas atividades, observamos que constituem-se de robôs construídos no Brasil. A alta porcentagem de robôs construídos (53.48\%), pode ser explicada devido aos altos custos de robôs importados como Lego Mindstorms. Entretanto, como descrito em [da Silva and Scherer 2012] nem todos os robôs construídos podem ser facilmente manipulados por alunos de ensino fundamental. Dentre os 23 trabalhos de robôs construídos, apenas 5 foram direcionados a alunos do ensino médio. Portanto, Lego Mindstorms ainda prevalece com 30\% dos robôs utilizados na RE dentre os especificados. Mais detalhes na Figura 5(a).

Com relação a locomoção dos robôs, apenas dois trabalhos não se enquadram em locomoção com rodas. Sendo um deles o robô humanoid NAO em [Pinto et al. 2014], e o segundo apresenta um robô aéreo autônomo não tripulado em [Nobre et al. 2006]. O baixo índice de robôs humanoides, aéreos ou aquáticos, é diretamente relacionado à sua alta complexidade de manipulação.

Na Figura 5(b) há uma ilustração da distribuição dos micro controladores utilizados nos robôs construídos. Ao discriminarmos regionalmente cada robô, foi notado um equilíbrio entre a região Nordeste e Sudeste com 11 trabalhos de robôs construídos para 
cada região. Dentre os 22 trabalhos avaliados, 5 da região Nordeste e 6 da região Sudeste são de robôs construídos através de arduino, mostrando a popularidade deste micro controlador.

\subsubsection{Q4 - Quantos trabalhos são reflexões, quantos são propostas e quantos apre- sentam experiências?}

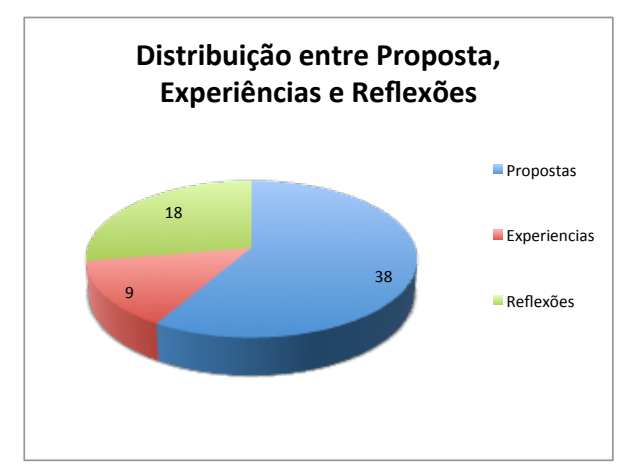

(a)

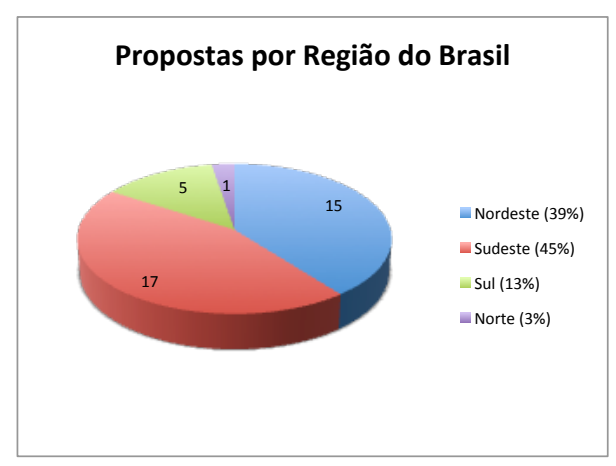

(b)

Figura 5. Propostas, Experiências e Reflexões. (a): Em Geral. (b): Propostas por Região.

Os trabalhos incluídos na RSL foram classificados entre: Propostas, Reflexões e Experiências. Os trabalhos identificados como Propostas, além de liderarem a pesquisa, são caracterizados por apresentar uma Proposta de metodologia ou um robô a serem aplicados na Experiência educacional. Os artigos incluídos em experiências apresentam uma abordagem a ser utilizada na RE com resultados explícitos. Por fim, nos trabalhos classificados em Reflexões há uma observação do autor a respeito da RE.

Através da distribuição regional das propostas, mais uma vez constatou-se um equilíbrio entre a região Nordeste que é responsável por 39\% das propostas, e a região Sudeste que abrange $45 \%$ delas. As demais regiões totalizam $18 \%$ do restante das propostas.

\section{Conclusão}

Neste trabalho, foi desvelado os resultados de uma revisão sistemática da literatura sobre o uso da robótica na educação publicados no período de 2004 e 2014 no Simpósio Brasileiro de Informática na Educação (SBIE), Workshop de Informática na Educação (WIE) e Workshop de Robótica na Educação (WRE). Para responder as quatro questões que nortearam a pesquisa, foram selecionados 65 artigos para esta revisão.

Com relação ao primeiro questionamento, pode-se notar que a maior parte das pesquisas está localizada nas regiões Sudeste e Nordeste do Brasil. Além disso, tem-se na região Nordeste a UFRN como principal instituição pesquisadora, enquanto nas demais regiões não encontramos uma discrepância acentuada entre as instituições com relação a quantidade de trabalhos publicados.

Referente ao segundo questionamento identificou-se a RE como uma matéria interdisciplinar, sendo utilizada por outras doze matérias sem incluir a própria robótica. A 
diferença entre a quantidade de artigos publicados nos contextos da educação fundamental e da educação nos níveis médio, superior e técnico mostrou que o uso da RE está tendo como alvo principal o ensino fundamental.

Para o terceiro questionamento, foi observado um grande número de kits de robótica construídos para o uso educacional, de forma a reduzir os custos, robôs construídos tendem a ser mais baratos que a utilização de robôs importados como Lego Mindstorms ou NAO. Dentre os robôs construídos, pôde-se destacar o uso do Arduino para a união e controle dos componentes robóticos. Com relação a locomoção apenas um robô humanoide e um robô aéreo fugiram da utilização de rodas como movimento.

Quanto ao quarto questionamento, de acordo com o cenários estudados foi observado um alto volume de propostas, principalmente de novos softwares, metodologias e robôs para utilização nos diversos níveis de ensino do país. Entretanto foi exposta uma escassez de experiências realizadas na área. Foi possível concluir que o ensino da robótica lida com muitas propostas fazendo dele uma ciência ainda muito teórica.

Como trabalhos futuros, pretendemos expandir esta revisão englobando outros importantes eventos de publicação na área da robôtica e/ou educação, tais como o Workshop de Educação em Computação (WEI), Simpósio Brasileiro de Automação Inteligente (SBAI), Revista Brasileira de Informática na Educação (RBIE) e Latin American Robotics Symposium (LARS).

\section{Referências}

Benitti, F. B. V., Vahldick, A., Urban, D. L., Krueger, M. L., and Halma, A. (2009). Experimentação com robótica educativa no ensino médio: ambiente, atividades e resultados. Workshop de Informática na Educação.

da Silva, F. I. and Scherer, D. (2012). A robótica móvel como instrumento de apoio à aprendizagem de computação. Workshop de Robótica na Educação.

d'Abreu, J. V. V. (2014). Robótica pedagógica: Percurso e perspectivas. Workshop de Robótica Educacional.

de Castro Pinto, M., da Fonseca Elia, M., and Sampaio, F. F. (2012). Formação de professores em robótica educacional com hardware livre arduino no contexto um computador por aluno. Workshop de Informática na Educação.

Kitchenham, B. (2007). Guidelines for performing systematic literature reviews in software engineering. EBSE Technical Report.

Monarcha, C. (1999). A escola normal da praça ? o lado noturno das luzes. Editora da Unicamp.

Nobre, J. C. S., Loubach, D. S., da Cunha, A. M., and Dias, L. A. V. (2006). Aprendizagem baseada em projeto (project-based learning - pbl) aplicada a software embarcado e de tempo real. Simpósio Brasileiro de Informática na Educação.

Papert, S. (1986). Logo: computadores e educação. Brasiliense.

Pinto, A. H. M., de Oliveira, L. O., Benicasa, A. X., Meneghetti, R. C. G., and Romero, R. A. F. (2014). Inserção de um robô humanoide no ensino de objetos geométricos $2 \mathrm{~d}$ sobrepostos. Simpósio Brasileiro de Informática na Educação. 